\title{
Palatal tremor in an atypical context
}

\author{
Arnaud Salami ${ }^{1 *}$, Nadine Yavo-Dosso ${ }^{2}$, Ababacar Diegane Faye ${ }^{1}$ and Assi Romaric Yapo ${ }^{1}$ \\ ${ }^{1}$ Department of Stomatology and Maxillofacial Surgery, Teaching Hospital of Treichville, Cote d'Ivoire \\ ${ }^{2}$ Department of ENT and Cervical Surgery, Teaching Hospital of Treichville, Abidjan, Côte d'Ivoire
}

\begin{abstract}
The literature concerning palatal tremor is probably poor because of their ignorance or rarity. Their clinical diagnosis is easy, and their aetiologies are various. The authors report a palatal tremor revealing a depressive syndrome.
\end{abstract}

\section{Introduction}

Palatal myoclonus and palatal tremor (PT) are the same entity corresponding to involuntary and rhythmic movements due to contractions of the muscles of the soft palate. They are poorly reported in the literature extremely rare and frequently ignored by head and neck specialists. Idiopathic forms are most common, represent a diagnosis of exclusion. Secondary PT are related to a traumatic, genetic, degenerative, infectious, immunological, vascular and even sometimes psychogenic pathologies [1]. We describe a case of PT revealing a depressive syndrome.

\section{Observation}

A previously healthy patient aged 34 was referred to the maxillofacial surgery department by an ENT doctor for a six-month history of referred otalgia. The anamnesis reported in addition to otalgia, tinnitus perceived as clicks little altering its daily activities. There were also apathy, insomnia, anorexia, anhedonia and anxiety. All these signs were consecutive to the discovery of a left ventricular hypertrophy during an annual check-up. The physical examination showed isolated and bilateral PT (about 60 contractions per minute) with brief rest periods (Video 1). Clicks responsible of tinnitus were audible. The exploration of the 12 pairs of cranial nerves was normal and no resting body tremor were noted. The cognitive functions and the rest of the examination

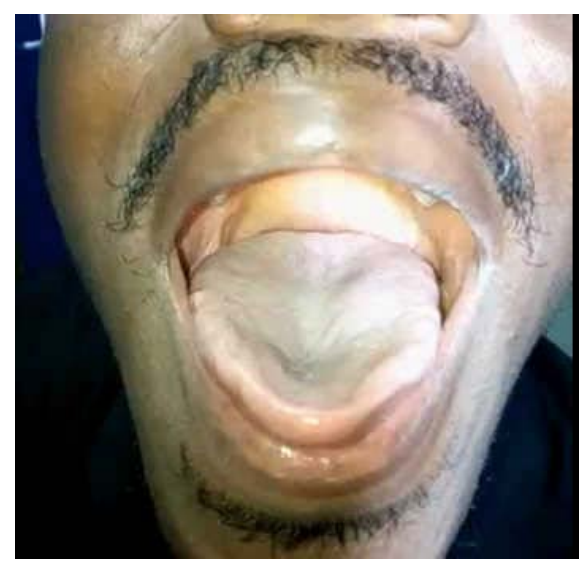

Video 1. Physical examination showing isolated and bilateral PT (about 60 contractions per minute) were normal. Magnetic resonance imaging (MRI) of the brain, tympanometry, and audiometry were normal. The diagnosis of MPP evolving in a depressive context was retained. PT and objective tinnitus gradually gone down after oral administration of Baclofen (5mg three times per day). The patient was referred for psychiatric consultation and adequate management of the depressive syndrome.

\section{Discussion}

PT correspond to the contraction of the tensor and levator veli palatini and may be an unusual objective sign that is part of the diagnosis of a depressive syndrome, but the pathogenesis is not known [2]. The presence of depressive syndrome can make treatment difficult, especially if the patient does not agree the proposed care protocol. In these cases, the management of the patient must include a neuropsychiatric component. Systematically, psychological care and eventual screening and treatment of depression should be realized in case of isolated PT. PT can be associated with other clinical signs of the ENT sphere such as tinnitus or otalgia [3]. Secondary PT in the context of depressive syndrome are very rare, especially when associated with objective tinnitus [2,4]. In this case report, objective tinnitus was characterized by clicks and explained by the contraction of the above-mentioned muscles. They can even maintain the depressive syndrome and in some cases push to suicide [5]. The use of Baclofen (benzodiazepine) significantly reduce the symptoms in this case. Other benzodiazepines such as clonazepam have also proven their effectiveness in the management of PT [2]. The treatment of PT can require medical procedures such as trans-palatal injection of botulinum toxin. Its effectiveness is proven but the effects die down after about 5 or 6 months, requiring repeated injections [5]. Before concluding to essential, non-organic PT, a cerebral MRI should be realized in search of impairment of the extra-pyramidal dentato-rubro-olivary pathways in the Guillain-Mollaret triangle [6].

${ }^{\star}$ Correspondence to: Arnaud Salami, Department of Stomatology and Maxillofacial Surgery, Teaching Hospital of Treichville, Abidjan, Cote d'Ivoire, Tel: +22507376024, E-mail: salami.arnaud@gmail.com

Key words: palatal tremor, depressive syndrome, objective tinnitus

Received: August 07, 2018; Accepted: August 24, 2018; Published: August 28 , 2018 


\section{Acknowledgements}

We would think Professor Konan Emmanuel who led this work and Professor Koffi-Aka Viviane for corrections.

\section{References}

1. Nagappa M, Bindu PS, Sinha S, Bharath RD, Sandhya M, et al. (2018) Palatal tremor revisited: disorder with nosological diversity and etiological heterogeneity. Can J Neurol Sci 45: 243-247. [Crossref]

2. Eberhardt O, Topka H (2017) Myoclonic Disorders. Brain Sci 7: 103.
3. Tatar EC, Gunay MM, Ocal B, Ozdek A, Korkmaz MH (2016) An electromyography guided botulinum toxin injection is effective treatment for objective tinnitus as an office procedure: A case report. Egyptian J Ear, Nose, Throat and Allied Sci 17: 159-161.

4. Guen A, Chalmin MC (2006) A propos de myoclonies associées à un syndrome dépressif. Rev Med Brux 27: 459-461.

5. Takahashi M, Kabaya K, Sekiya K, Matsuda F, Sekiya Y, et al. (2017) An improved system for grading and treating tinnitus. Auris Nasus Larynx 45: 711-717. [Crossref]

6. Salazar R, Miller D (2013) Symptomatic palatal tremor of cortical origin due to stroke. J Clin Neurosci 20: 757-759. [Crossref]

Copyright: $(02018$ Salami A. This is an open-access article distributed under the terms of the Creative Commons Attribution License, which permits unrestricted use, distribution, and reproduction in any medium, provided the original author and source are credited. 